\title{
Power law relationship between parameters of earthquakes and precursory electrical phenomena
}

\author{
E. Dologlou \\ Solid State Section, Department of Physics, University of Athens, Panepistimiopolis, Zografos, 15784 Athens, Greece
}

Received: 27 May 2008 - Revised: 24 July 2008 - Accepted: 28 July 2008 - Published: 2 September 2008

\begin{abstract}
A number of Seismic Electric signals data have been accumulated during the last two decades that also includes the signals observed before the magnitude 6 class earthquakes that occurred in Greece very recently i.e., the first two months of 2008.

Using all the available data we investigate whether a possible interconnection exists between the lead time of Seismic Electric Signals and the stress drop of subsequent earthquakes. We show that for "non thrust" earthquakes a power law relation emerges with an exponent value around 0.29 , the possible physical meaning of which is discussed. This value is very close to the range of critical exponents that govern the fracture processes and is also comparable with the value of the slope, found much earlier by the VAN group, in the linear relation between the logarithm of the SES amplitude and the earthquake magnitude.
\end{abstract}

\section{Introduction}

There is a tendency to consider that electromagnetic phenomena related to earthquake preparation processes in the highly heterogeneous crust should be described by "non linear" models which obey power laws and have fractal geometry (Varotsos, 2005). Pop science strongly supports the concept that rupture in non homogeneous media, percolation and earthquakes belong to critical phenomena.

The scope of the present paper is to treat pre-seismic electromagnetic signals and associated earthquakes within the framework of "critical point" theories and to investigate possible power law relationships with fractal critical exponents between their parameters.
Precursory changes of the earth's electrotelluric field in the past 25 years have been continuously monitored at different sites in Greece (Varotsos and Alexopoulos, 1984a, b; Varotsos et al., 1986). Transient, low frequency $(<1 \mathrm{~Hz})$ changes in this field, the so called Seismic Electric Signals (SES), have been used by the VAN group for earthquake forecasting. Some interesting characteristics of the SES signals (Varotsos and Alexopoulos, 1984a, b, 1987; Varotsos et al., 1993) are: i) the lead time, $\Delta t$, which is the time difference between the SES detection and the earthquake occurrence and can vary from a few hours to a few months. ii) the selectivity effect. That means that SES signals can be detected at "preferred" sites which can be sensitive to some specific seismic areas at long distances and inactive to other areas at closer distances iii) the relation, for the same measuring site, between the amplitude of the SES and the magnitude $M$ of the subsequent earthquake according to the experimental formula:

$$
\log E=\alpha M+b
$$

where $E=\Delta V / L$ is the SES amplitude ( $\Delta V$ is the potential change measured by a dipole of length $L$ ), a $\simeq 0.3-0.4$ and $b$ is a site-dependent constant. This relation was found to be (Varotsos and Alexopoulos, 1984a, b) valid for all measuring sites and corresponding pre-seismic regions.

A variety of models have been subsequently proposed for the explanation of SES generation (e.g., see chapter 12 of Varotsos, 2005). Below, we restrict ourselves to a summary of two of them, i.e., the piezostimulated currents (PSC) (Varotsos and Alexopoulos, 1986) and the electrokinetic model (Mizutani et al., 1976; Ishido and Mizutani, 1981; Gershenzon and Gokhberg, 1993; Yoshida et al., 1998; Surkov et al., 2002) that are relevant to the present study.

\section{Correspondence to: E. Dologlou}

(edologl@phys.uoa.gr)

Published by Copernicus Publications on behalf of the European Geosciences Union. 


\section{Pressure stimulated current (PSC) model}

When aliovalent impurities are introduced into an ionic solid a number of vacancies appear for reasons of charge compensation (Varotsos and Mourikis, 1974; Kostopoulos et al., 1975). A portion of these vacancies are attracted by the near by impurities and form electric dipoles (Varotsos and Miliotis, 1974) which under isothermal conditions, as a result of increase or decrease of pressure, can change the time needed for their orientation, giving rise to a transient current, called piezostimulated current (PSC). The relaxation time, $\tau$, of these dipoles is given by the formula

$\tau=(\lambda \nu)^{-1} \exp (g / k T)$

where $T$ is the temperature, $\lambda$ the number of jumpable and neighboring paths for the migration with an attempt frequency $v$ and $g$ the Gibbs energy for the orientation process. Before an earthquake, the stress (pressure) gradually increases in the candidate focal area with a rate $b(=d P / d t)$. A change of pressure affects in general not only some bulk properties, e.g., the compressibility (Varotsos and Alexopoulos, 1980a; Varotsos, 1981), but also the thermodynamic parameters for the formation (Varotsos, 1977), migration (Varotsos and Alexopoulos, 1978) and activation (Lazaridou et al., 1985; Varotsos and Alexopoulos, 1980b, c) of defects in solids. If the migration volume $v=(d g / d P)_{T}$ or the activation is negative, an increase of pressure, $P$, leads to a decrease of the relaxation time $\tau$. When a solid is under the initial pressure the relaxation time may be very long but as the pressure increases, the relaxation time becomes gradually smaller. It can be shown (Varotsos and Alexopoulos, 1986) that when the pressure reaches a critical value, $P_{\mathrm{cr}}$, a transient current, due to a cooperative orientation of dipoles, is emitted and the following relation holds:

$$
\frac{b v}{k T}=-\frac{1}{\tau\left(P_{\mathrm{cr}}\right)}
$$

where $\tau\left(P_{\mathrm{cr}}\right)$ is the value of relaxation time at $P=P_{\mathrm{cr}}$. When the pressure (stress) still continues to increase, it finally reaches the fracture stress $P_{\mathrm{fr}}$ where the failure of the solid occurs. Therefore, the emission of this transient current (e.g., SES) can be considered as a precursor to the failure of a solid (e.g., earthquake). The lead time $\Delta t$ between the emission of the SES and the impending earthquake is given by

$\Delta t=\left(P_{\mathrm{fr}}-P_{\mathrm{cr}}\right) / b$

where $P_{\mathrm{fr}}$ and $P_{\mathrm{cr}}$ are the critical values of pressure for the solid failure and SES emission, respectively.

\section{Electrokinetic model}

Another plausible model for the SES generation is the Electrokinetic effect - or streaming potential - (Dobrovolsky et al., 1989; Mizutani et al., 1976; Ishido and Mizutani, 1981;
Yoshida et al., 1998). In a porous or fractured medium a double electric layer is formed between the solid-liquid interface.. This double layer consists of ions trapped in the solid phase, with an equivalent amount of ionic charge of the opposite sign distributed in the liquid phase near the boundary. As the fluid flows through the medium, a potential caused by the relative motion between the solid and liquid., known as streaming potential, arises across the rock. Under a pressure gradient the fluid flows and the carried charges move to the direction of the flow generating an electrical current. According to Surkov et al. (2002) the electrokinetic effect exhibits fractal properties and fractal critical exponents. They made the hypothesis that the focal area of an impending earthquake includes water-saturated porous rocks with fluid-filled pore channels. During the pre-earthquake stage a number of fresh cracks will appear in the vicinity of the future hypocenter forming a fracture zone. The scale of a fracture zone may vary from hundreds of meters up to several kilometres. Most of the fresh cracks, being under lower pressure due to cracking, are filled with water which penetrates into them from the uncracked outer region as soon as a network of connected channels or fractal clusters is formed. It was assumed that the pore space in the fracture zone exhibits fractal structure. Surkov et al. (2002) supposed that the porosity $n$, after the cluster formation, decreases from the center of the fracture zone towards the periphery by a certain law. The percolation threshold $n_{c}$ is exceeded only in the internal area with typical size $L$. Fractal properties near the threshold are determined by the correlation length

$\xi=\frac{1}{\left|n-n_{c}\right|^{v}}$

where $\nu=0.88$ is the correlation length critical exponent (Stauffer, 1979). The fractal region occupies a spherical shell of thickness $H$ which near the distance $r=L$ can be presented (Surkov, 2002) by:

$H \sim\left(\frac{d n}{d r}\right)^{\left(-\frac{v}{v+1}\right)} \sim L^{v /(v+1)}$

According to percolation theory, the rock conductivity $\sigma_{r}$ depends on the porosity $n$ as:

$\sigma_{r} \sim\left(n-n_{c}\right)^{t}$

where $t \sim 1.6$ is the transport critical exponent (Stauffer, 1979). Surkov et al. (2002) concluded that the electrokinetic current density $p_{f}$, from the external fractal zone is given by:

$p_{f} \sim L^{1-(t-v) /(1+v)}$

which with $t=1.6$ and $\nu=0.88$ leads to:

$p_{f} \sim L^{0.5}$

If the SES signal results from the electrokinetic current generated in the external fractal zone of the preseismic volume, the SES amplitude $E$ must be proportional to the amplitude 
$p_{f}$. Thus, since $E \sim p_{f}$ upon considering Eqs. (8) and (9), Surkov et al. (2002) finally obtained a relation compatible to VAN experimental data:

$$
\log E=\alpha M+b, \alpha=0.5\left(1-\frac{t-v}{1+v}\right) \approx 0.31
$$

The above equation indicates that only when we consider that the candidate fracture region has fractal geometry, the value of $\alpha$ do come very close to the experimental one observed by Varotsos and Alexopoulos (1984a). That possibly means that the emission of the SES starts when the pre-focal area enters the critical regime and consequently exhibits fractal geometry. The picture of similar critical exponents in the electrokinetic effect and the much earlier observed SES probably implies that the same underlying physics govern both phenomena. Thus, irrespective of the considered model it seems that probably SES is emitted upon approaching criticality and hence fractal properties and power laws should prevail (e.g., see Varotsos et al., 2004; Varotsos, 2005). In the view of this expectation the main goal of this paper is to search for preliminary relations between the lead time of the SES signals and the stress drop of the impending earthquakes and to examine whether they follow power law behavior with exponents falling in the range of critical values.

\section{Stress drop}

An important scaling source parameter of an earthquake is the stress drop, $\Delta \sigma$ (e.g., Brune, 1970; Hanks and Wyss, 1972; Boatwright, 1980; Anderson, 1997) which in its original definition, is the difference between two states of stress at a point on a fault before and after rupture (Kanamori and Anderson, 1975). Stress drop parameter can be obtained from teleseismic body waves (Hanks and Wyss, 1972; Molnar and Wyss, 1972; among others) by using different models of which the most popular are Brune's and Madariaga's models (Brune, 1970, 1971; Madariaga, 1976). In the present work we refer to the Brune's model according to which the source displacement spectra of the far field body waves (teleseismic $\mathrm{P}$ - and S-waves) are used to obtain the Brune's stress drop, $\Delta \sigma_{B}$, through the equation:

$\Delta \sigma_{B}=0.44 M_{o} / r^{3}$

where $M_{o}$ is the seismic moment and $r$ the source radius when a circular fault is assumed. The seismic moment is estimated as:

$M_{0}=\frac{\left(4 \pi \rho V^{3} \Omega_{0} R\right)}{\left(k R_{\theta \varphi}\right)}$

where $\rho$ is the density of the medium, $R$ is the hypocentral distance between the source and the receiver, $V$ is the $\mathrm{P}$ or $\mathrm{S}$-wave velocity near the source, $\Omega_{o}$ is the low frequency spectral level derived from $\mathrm{P}$-waves or S-waves, respectively.
The factor $k$ is the free surface operator and $R_{\theta \phi}$ is the average radiation pattern coefficient. Finally, source radius is computed as

$r=0.37 \mathrm{~V} / f_{o}$

where $f_{o}$ is the spectral corner frequency, defined from the intersection of the low level $\Omega_{o}$ and the fall off in the displacement spectra of $\mathrm{P}$ - or S-waves, respectively.

\section{Data and analysis}

In the present work we deal with all large earthquakes with available stress drop values which occurred in Greece and were preceded by a SES signal for the period 19812008. We found 12 earthquakes with reported Brune's stress drop values derived from teleseismic body waves spectra (Papazachos et al., 1988; Stavrakakis and Blionas, 1990; Chouliaras and Stavrakakis, 1997; Stavrakakis et al., 2002; Benetatos et al., 2002; Papadimitriou, personal communication, 2007, 2008) and two earthquakes with reported fault dimensions and seismic moments (Kiratzi et al., 1985). The Brune's stress drop values for these two events (no. 1 and 2) have been calculated with the aid of Eq. (11). We used the seismic moments $M o_{1}=2.4 \times 10^{26} \mathrm{dyn} \mathrm{cm}$ and $M o_{2}=9.4 \times 10^{25} \mathrm{dyn} \mathrm{cm}$, given by Kiratzi et al. (1985), for events no. 1 and 2 , respectively. The corresponding radii $r_{1}$ and $r_{2}$ for a circular fault in Eq. (11), were obtained from the reported (Kiratzi et al., 1985) fault surfaces $S_{1}=1620 \mathrm{~km}^{2}$ and $S_{2}=784 \mathrm{~km}^{2}$ according to the relations $S_{1}=\pi r_{1}^{2}$ and $S_{2}=\pi r_{2}^{2}$ and were found to be $r_{1}=22.7 \mathrm{~km}$ and $r_{2}=15.8 \mathrm{~km}$. The derived, from Eq. (11), Brune's stress drop values (9 bars and 10.5 bars) for events no. 1 and 2 , respectively, are given in Table 1. The value of 9 bars for event no. 1 is compatible with the one (i.e., 8 bars) estimated for a fault surface determined by aftershocks (Kiratzi et al., 1991).

Additional information concerning the focal mechanism (strike-slip, normal or thrust type) is also given for all studied events by Harvard CMT (Centroid Moment Tensor solutions) - when available - or EMSC (European-Mediterranean Seismological Center) solutions (e.g. Dziewonski et al., 1995, and references therein http://neic.usgs.gov/neis/sopar/ and http://www.emsc-csem.org).

The associated SES signals were detected at various stations of the telemetric VAN network. The lead times, $\Delta t$, for events no. 1, 2 are reported by Varotsos and Alexopoulos (1984a) - see their Figs. 3 and 2, respectively - while for events no. 3-10 by Varotsos (2005). The three recent events (no. 11-13) are given by Varotsos et al. (2007), for the event no. 11 and Varotsos et al. (2008) for no. 12, 13.

All thirteen earthquakes numbered in chronological order along with their seismic parameters (dates, epicentres, depths, moment magnitudes $M_{w}$ ), Harvard CMT source mechanism (strike-slip, normal or thrust type) and SES parameters (station of detection and lead time), are listed in 
Table 1. All 13 earthquakes with available stress drop values and precursory SES signals in Greece from 1981 to 2008 , along with their dates, epicentres, depths, moment magnitudes $M_{w}$, stress drop values $\left(\Delta \sigma_{B}\right)$, SES station, SES lead times $\Delta t$ (see text) and mechanism type (strike-slip, normal or thrust). Events are numbered in chronological order and References for the stress drop values are given in the last column.

\begin{tabular}{|c|c|c|c|c|c|c|c|c|c|c|c|c|c|}
\hline$n$ & yy mm dd & $H$ & Min & $S$ & Lat & Long & $\begin{array}{l}\text { Depth } \\
(\mathrm{km})\end{array}$ & $M w$ & $\begin{array}{l}\Delta \sigma_{B} \\
\text { bars }\end{array}$ & $\begin{array}{l}\text { SES } \\
\text { station }\end{array}$ & $\begin{array}{l}\text { SES } \\
\Delta t \text { days }\end{array}$ & $\begin{array}{l}\text { Source } \\
\text { mechanism }\end{array}$ & References \\
\hline 1 & 811219 & 14 & 10 & 50.7 & 39.24 & 25.23 & 12 & 6.8 & 9,01 & PIR & 0.3 & strike - slip & see text \\
\hline 2 & 820118 & 19 & 27 & 24.5 & 40 & 24.32 & 6 & 6.6 & 10,5 & PIR & 0.3 & strike - slip & see text \\
\hline 3 & 830117 & 12 & 41 & 29 & 38.09 & 20.19 & 10 & 6.9 & 14,0 & PIR & 1.8 & strike + thrust & Stavrakakis and Blionas (1990) \\
\hline 4 & 860913 & 17 & 24 & 34 & 37.03 & 22.2 & 15 & 5.9 & 5,0 & KER & 5 & normal & Papazachos et al. (1988) \\
\hline 5 & 950504 & 0 & 34 & 11 & 40.54 & 23.63 & 15 & 5.4 & 2,5 & ASS & 28.5 & normal & Chouliaras and Stavrakakis (1997) \\
\hline 6 & 950513 & 8 & 47 & 15 & 40.16 & 21.67 & 15 & 6.5 & 6,3 & IOA & 25.5 & normal & Chouliaras and Stavrakakis (1997) \\
\hline 7 & 950615 & 0 & 15 & 56 & 38.1 & 22.46 & 15 & 6.5 & 2,9 & VOL & 46 & normal & Chouliaras and Stavrakakis (1997) \\
\hline 8 & 990907 & 11 & 56 & 56 & 37.97 & 23.6 & 15 & 6 & 3,0 & LAM & 6 & normal & Stavrakakis and Chouliaras (2002) \\
\hline 9 & 010726 & 0 & 21 & 44 & 38.96 & 24.29 & 15 & 6.5 & 9,0 & PIR & 130 & strike - slip & Benetatos et al. (2002) \\
\hline 10 & 030814 & 5 & 15 & 8 & 38.7 & 20.67 & 15 & 6.3 & 8,0 & PIR & 6 & strike - slip & Papadimitriou (2007) \\
\hline 11 & 080106 & 5 & 14 & 20 & 37.22 & 22.69 & 75 & 6.2 & 9,0 & PAT & 60 & thrust + strike & Papadimitriou (2008) \\
\hline 12 & 080204 & 20 & 25 & 9.5 & 38.08 & 21.94 & 20 & 5 & 1,6 & PAT & 25 & strike - slip & Papadimitriou (2008) \\
\hline 13 & 080214 & 10 & 9 & 22.7 & 36.5 & 21.67 & 29 & 6.9 & 9,0 & PIR & 30 & thrust & Papadimitriou (2008) \\
\hline
\end{tabular}

Table 1. Bibliographical references for the stress drop values are given in the last column of this Table. A map of Greece with the epicenters (denoted by stars) of all thirteen earthquakes along with their focal mechanism (attached numbers refer to the event number) and the position of the SES stations (presented by squares) is shown in Fig. 1.

From Table 1 we observe that among thirteen studied earthquakes only two events (no. 11 and no. 13) have a source mechanism of thrust type. Geodynamics of thrust mechanism are quite different from those of other mechanisms (i.e., normal, strike-slip). Earthquakes of thrust type usually occur in collision or subduction zones which are characterized by high accumulation of strain. Another notable remark is the unusual long lead time (i.e., $\Delta t=130$ days) of event no. 9 which could be attributed to specific geodynamics (e.g., very small crustal thickness, upwelling of mantle) that prevail in the pre-seismic region. This case is thoroughly discussed by Dologlou et al. (2008) and thus event no. 9 will be excluded from this study.

The possibility of existence of any relationship between the stress drop of the earthquakes and the lead time of the SES signals has been examined by plotting $\Delta \sigma_{B}$ versus $\Delta t$ as depicted in the diagram of Fig. 2. A linear or power law fitting applied to all remaining twelve events does not yield any meaningful relation. On the contrary, when taking into account only the "non thrust" earthquakes, a power law correlation seems to emerge and the corresponding equation and correlation coefficient $R_{P}$ (power) are shown on the top of the diagram in Fig. 2. Different symbols on this plot as dots, crosses and triangles represent events with normal, strike-slip and thrust mechanism, respectively.

\section{Discussion}

In recent years, fracture of solids and consequently generation of earthquakes are treated under the new prospective of critical phenomena.

At the beginning of eighties, field observations showed, as mentioned above, the experimental relation given by Eq. (1) which could imply that interconnection between seismological and precursory electromagnetic phenomena exhibits power law behavior. This relation (Eq. 1) having a slope $\alpha \simeq 0.3-0.4$ was considered at that time as an experimental result associated with a common geometry of the emitting sources (future focal areas). Actually, this turned out later to be the fractal geometry discussed in various critical phenomena like fracture in the case of earthquakes.

Several efforts, summarized in Varotsos (2005), have been made to interconnect the value $\alpha \simeq 0.3-0.4$ with the critical exponents such as the above mentioned by Surkov et al. (2002).

Sornette et al. (1989) also attempted a physical insight of the value of this slope by interconnecting it with critical exponents emerged in the crack percolation theory. This attempt (Sornette and Sornette, 1990) is summarized below.

The energy $W$ released by an earthquake at the source is related to the magnitude $M$ through the formula (Kanamori and Boschi, 1983):

$\log W=\delta M+\gamma$

where $\delta \approx 1$ for $M \leq 7$. From Eqs. (10) and (14) the following scaling law derives:

$E=\Delta V / L=W^{\alpha / \delta}$

where $\alpha=0.3-0.4$ and $\delta \approx 1$ and thus the exponent $\alpha / \delta$ should lie between 0.3 and 0.4 . 


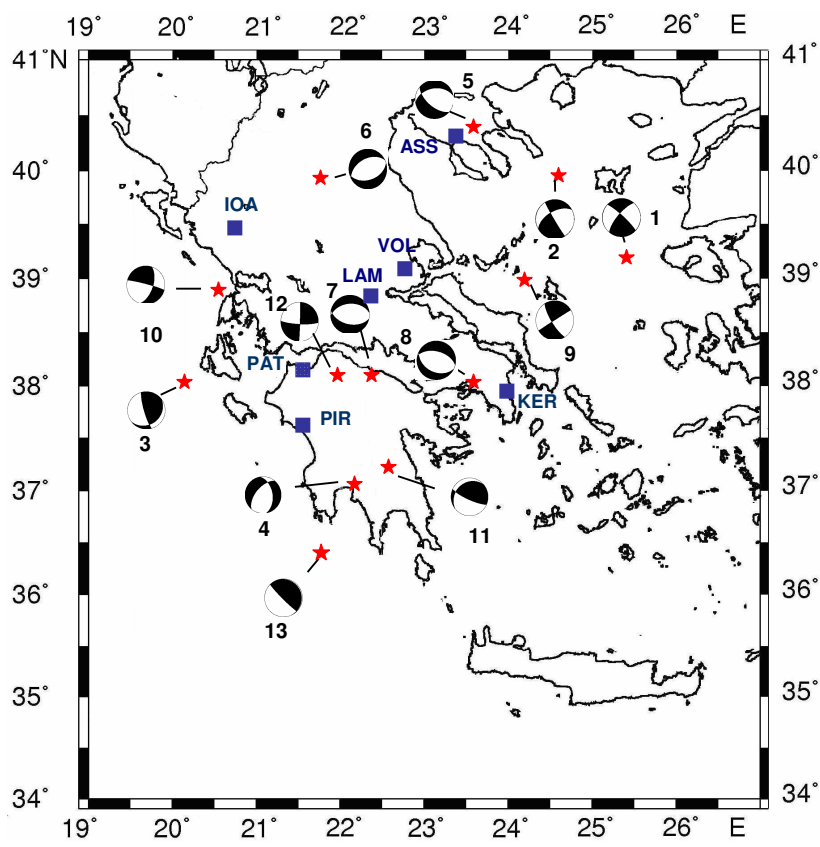

Fig. 1. Map of Greece with all epicenters (denoted by stars) for the 13 earthquakes listed in Table 1, during the period 1981-2008, along with their CMT fault plane solutions. A lower hemisphere projection is used with black and white quadrants (beach balls) for compression and dilatation respectively. Numbers attached refer to the events in Table 1 and squares denote the position of SES stations.

According to Sornette et al. (1989) the dependence with respect to strain, $\varepsilon$, of the piezoelectric coupling between the strain and the electric displacement, $D$, at constant applied stress, $\sigma$, is given by:

$(\partial \varepsilon / \partial D)_{\sigma} \approx \varepsilon^{\nu(d-1) / \tau}$

where $d$ stands for the space dimension. Note that $D$ is interrelated with the electric field through the static dielectric constant, which varies upon increasing pressure due mainly to the change of the interatomic distance that markedly affects the ionic polarizability (Varotsos, 1978) but not the electronic polarizability (Varotsos, 1980). The values of $v$ and $\tau$ exponents, estimated from crack percolation theory, are $\nu=4 / 3$ and $\tau=3.0$ for $d=2$ (two dimensional case) and $\nu=0.9$ and $\tau=3.8$ for $d=3$ (three dimensional case). Considering that $W=\sigma \varepsilon$, at constant stress $\sigma$, the exponents in the right side of Eqs. (15) and (16) should be equal. From the above mentioned values for $v, \tau$ and $d$ the quantity $v(d-1) / \tau=1 / 3$ or 0.47 for $d=2$ and $d=3$, respectively. This is in rather good agreement with the value $\alpha / \delta=0.3-0.4$ derived from the experimental relation between the logarithm of SES amplitude and the earthquake magnitude.

Thus, Sornette et al. (1989) suggested that power law relationships (see Eq. 16) prevail in the fracture processes with critical exponent values 0.33 and 0.47 for the case of 2 or 3

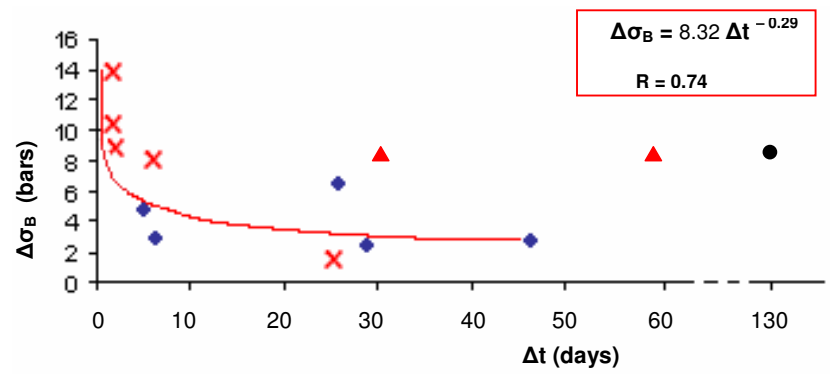

Fig. 2. Plot of the Brune stress drop, based on far field body wave source spectra $\Delta \sigma_{B}$ against the lead time $\Delta t$. Crosses $\times$, dots $\bullet$ and triangles $\Delta$ indicate earthquakes of strike-slip, normal and the thrust type, respectively. The solid circle refers to the event no. 9 which is excluded from this study for reasons explained in the text. A power law fitting has been applied to all remaining "non thrust" events and the derived equation along with the corresponding correlation coefficient $R_{P}$ is displayed on the top of the diagram.

dimensions, respectively. In addition, Surkov et al. (2002), as mentioned in the Introduction, suggested that the Electrokinetic effect also exhibits critical behavior in the future fracture zone, which is expressed by power law equations (Eqs. 6 and 8). The values of the two critical exponents related to this effect, namely the correlation length critical exponent $\nu=0.88$ and the transport critical exponent $t \sim 1.6$ according to Eq. (10) support the idea that may be SES emission emerges when the focal area enters the critical stage.

In the present paper the investigation of a possible correlation between the stress drop, $\Delta \sigma_{B}$, of the earthquake and the lead time, $\Delta t$, of the precursory SES leads to a power law relation given by the formula:

$\Delta \sigma_{B}=8.32 \Delta t^{-0.29}$

with an exponent value of 0.29 which is very close to the lower limit of the range 0.3-0.4 for critical exponents for fracture. This result, obtained from different parameters of SES and earthquakes, constitutes an independent preliminary indication that SES and earthquakes preparation processes could probably be governed by similar physics.

\section{Conclusion}

Possible interconnection between SES lead time and earthquake stress drop has been examined. A power law relation results with an exponent around 0.29 which lies very close to the range of critical exponents expected by various models suggested for fracture. In addition, this value $\approx 0.3$ is comparable to the one reported by Varotsos and Alexopoulos (1984a, b), for the slope of the linear relation between the logarithm of SES amplitude and earthquake magnitude. Thus, the present preliminary results could be considered as an implication that may be the same underlying physics is responsible for SES generation and earthquake preparation process. In order to confirm the above findings and get 
robust conclusion, new data from future major earthquakes are required.

Edited by: M. Contadakis

Reviewed by: two anonymous referees

\section{References}

Anderson, J. G.: Seismic energy and stress drop parameters for a composite source model, B. Seismol. Soc. Am., 87, 85-96, 1997.

Benetatos, C., Roumelioti, Z., Kiratzi, A., and Melis, N.: Source parameters of the M 6.5 Skyros island (North Aegean Sea) earthquake of July 26, 2001, Ann. Geophys.-Italy, 45, 513-526, 2002.

Boatwright, J.: A spectral theory for circular seismic sources: simple estimates of dimension, dynamic stress drop and radiated seismic energy, B. Seismol. Soc. Am., 70, 1-27, 1980.

Brune, J.: Tectonic stress and the spectra of seismic shear waves, J. Geophys. Res., 75, 4997-5009, 1970.

Brune, J. N.: Correction, J. Geophys. Res., 76, p. 5002, 1971.

Chouliaras, G. and Stavrakakis, G.: Seismic source parameters from a new dial-up seismological network in Greece, Pure Appl. Geophys., 150, 91-111, 1997.

Dobrovolsky, I., Gershenzon, N., and Gokhberg, M.: Theory of electrokinetic effects occurring at the final stage in the preparation of a tectonic earthquake, Phys. Earth Planet. Inter., 57(1-2), 144-156, 1989.

Dologlou, E.: Possible relationship between Seismic Electric Signals (SES) lead time and earthquake stress drop, Proc. Jpn. Acad. Ser. B, 84, 117-122, 2008.

Dologlou, E., Hadjicontis, V., and Mavromatou, C.: Electrical precursors of earthquakes in Aegean Sea during the last decade (1997-2007), Nat. Hazards Earth Syst. Sci., 8, 123-128, 2008, http://www.nat-hazards-earth-syst-sci.net/8/123/2008/.

Dziewonski, A. M., Ekström, G., and Salganik, M. P.: CentroidMoment Tensor Solutions For October-December 1994, Phys. Earth Planet. Inter., 91, 187-201, also available at: http://neic. usgs.gov/neis/sopar/, 1995.

Gershenzon, N. and Gokhberg, M.: On the origin of electrotelluric disturbances prior to an earthquake in Kalamata, Greece, Tectonophysics, 224, 169-174, 1993.

Hanks, T. and Wyss, M.: The use of body wave spectra in the determination of seismic source parameters, B. Seismol. Soc. Am., 62, 561-589, 1972.

Ishido, T. and Mizutani, H.: Experimental and theoretical basis of electrokinetic phenomena in rock-water systems and its applications to geophysics, J. Geophys. Res., 86, 1763-1775, 1981.

Kanamori, H. and Anderson, D. L.: Theoretical basis of some empirical relations in seismology, B. Seismol. Soc. Am., 65, 10731095, 1975.

Kanamori, H. and Boschi, E. (Eds.): Earthquakes, Observation, Theory and Interpretation, North Holland, Amsterdam, 1983.

Kiratzi, A. A., Wagner, G. S., and Langston, C. A.: Source Parameters of Some Large Earthquakes in Nortern Aegean Determined by Body Waveform Inversion, PAGEOPH, 135, 515-527, 1991.

Kiratzi, A. A., Karakaisis, G. F., Papadimitriou, E. E., and Papazachos, B. C.: Seismic-Source Parameter Relations for Earthquakes in Greece, PAGEOPH, 123, 27-41, 1985.

Kostopoulos, D., Varotsos, P., and Mourikis, S.: Conductivity of crystalline NaI, Can. J. Phys., 53, 1318-1320, 1975.
Lazaridou, M., Varotsos, C., Alexopoulos, K., and Varotsos, P.: Point defect parameters of LiF, J. Phys. C: Solid State, 18, 38913895, 1985.

Madariaga, R.: Dynamics of an expanding circular fault, B. Seismol. Soc. Am., 66, 639-666, 1976.

Mizutani, H., Ishido, T., Yokokura, T., and Ohnishi, S.: Electrokinetic phenomena associated with earthquakes, Geophys. Res. Lett., 3, 365-368, 1976.

Molnar, P. and Wyss, M.: Moments, Source Dimensions and Stress Drops of Shallow Focus Earthquakes in the Tonga-Kermades Arc, Physics Earth Planet. Interiors, 6, 263-278, 1972.

Papazachos, B., Kiratzi, A., Karakostas, B., Panagiotopoulos, D., Scordilis, E., and Mountrakis, D.: Surface Fault Traces, Fault Plane Solution and Spatial Distribution of the Aftershocks of the September 13, 1986 Earthquake of Kalamata (Southern Greece), PAGEOPH, 126, 55-68, 1988.

Sarlis, N., Lazaridou, M., Kapiris, P., and Varotsos, P.: Numerical model of the selectivity effect and the $\Delta \mathrm{V} / \mathrm{L}$ criterion, Geophys. Res. Lett., 26, 3245-3248, 1999.

Sornette, D., Lagier, M., Roux, S., and Hansen, A.: Critical piezoelectricity in percolation, J. Phys.-Paris, 50, 2201-2216, 1989.

Sornette, A. and Sornette, D.: Earthquake rupture as a critical point: consequences for telluric precursors, Tectonophysics, 179, 327334, 1990.

Stavrakakis, G. and Blionas, S.: Eastern Mediterranean Region Based on an Iterative Maximum Entropy Technique, PAGEOPH, 132, 679-698, 1990.

Stavrakakis, G., Chouliaras, G., and Panopoulou, G.: Seismic Source Parameters for the ML=5.4 Athens Earthquake (7 September 1999) from a New Telemetric Broad Band Seismological Network in Greece, Natural Hazards, 27, 47-60, 2002.

Stauffer, D.: Scaling theory of percolation clusters, Phys. Reports, 54(1), 1-74, 1979.

Surkov, V., Uyeda, S., Tanaka, H., and Hayakawa, M.: Fractal Properties of medium and seismoelectric phenomena, J. Geodynamics, 33, 477-487, 2002.

Varotsos, P. A.: On the temperature and pressure dependence of the defect formation volume in ionic crystals, J. Phys. (France) Lett., 38, L455-L458, 1977.

Varotsos, P. A.: An estimate of the pressure dependence of the dielectric constant in alkali halides, Phys. Status Solidi B, 90, 339343, 1978.

Varotsos, P.: Determination of the dielectric constant of alkali halide mixed crystals, Phys. Status Solidi B, 100, K133-138, 1980.

Varotsos, P.: Determination of the composition of the maximum conductivity or diffusivity in mixed alkali halides, J. Phys. Chem. Solids, 42, 405-407, 1981.

Varotsos, P. A.: The Physics of Seismic Electric Signals, Terra Pub. Tokyo, ISBN 4-88704-136-5, 2005.

Varotsos, P. A. and Mourikis, S.: Difference in conductivity between LiD and LiH crystals, Phys. Rev. B, 10, 5220-5224, 1974.

Varotsos, P. and Miliotis, D.: New aspects on the dielectric properties of the alkali halides with divalent impurities, J. Phys. Chem. Solids, 35, 927-930, 1974.

Varotsos, P. and Alexopoulos, K.: Calculation of the migration volume of vacancies in ionic solids from macroscopic parameters, Phys. Status Solidi A, 47, K133-136, 1978.

Varotsos, P. and Alexopoulos, K.: Prediction of the compressibility of mixed alkali halides, J. Phys. Chem. Solids, 41, 1291-1294, 
1980a.

Varotsos, P. and Alexopoulos, K.: Calculation of diffusion coefficients at any temperature and pressure from a single measurement. I. Self diffusion, Phys. Rev. B, 22, 3130-3134, 1980 b.

Varotsos, P. and Alexopoulos, K.: Negative activation volumes of defects in solids, Phys. Rev. B, 21, 4898-4899, 1980c.

Varotsos, P. and Alexopoulos, K.: Physical properties of the variations of the electric field of the earth preceding earthquakes, I, Tectonophysics, 110, 73-98, 1984a.

Varotsos, P. and Alexopoulos, K.: Physical properties of the variations of the electric field of the earth preceding earthquakes, II. Determination of epicentre and magnitude, Tectonophysics, 110, 99-125, 1984b.

Varotsos, P. and Alexopoulos, K.: Thermodynamics of Point Defects and their relation with the bulk properties, edited by: Amelinckx, S., Gevers, R., and Nihoul, J., North Holland, Amsterdam, 1986.

Varotsos, P. and Alexopoulos, K.: Physical properties of the variations in the electric field of the earth preceding earthquakes, III, Tectonophysics, 136, 335-339, 1987.

Varotsos, P., Alexopoulos, K., and Lazaridou, M.: Latest aspects of earthquake earthquake prediction in Greece based on Seismic Electric Signals II, Tectonophysics, 224, 1-37, 1993.
Varotsos, P., Sarlis, N., and Skordas, E.: Seismic Electric Signals and 1/f noise in natural time, arXiv:cond mat: 0711.3766v1, 23 November 2007.

Varotsos, P., Sarlis, N., and Skordas, E.: Seismic Electric Signals and 1/f noise in natural time, arXiv:cond mat: 0711.3766v3, 1 February 2008.

Varotsos, P., Alexopoulos, K., Nomicos, K., and Lazaridou, M.: Earthquake prediction and electric signals, Nature, 322, 120, 1986.

Varotsos, P. A., Sarlis, N. V., Skordas, E. S., and Lazaridou, M. S.: Entropy in the natural time-domain, Phys. Rev. E, 70, 011106, 2004.

Uyeda, S., Khaled Al-Damegh, S., Dologlou, E., and Nagao, T.: Some relationship between VAN seismic electric signals (SES) and earthquake parameters, Tectonophysics, 304, 41-55, 1999.

Wyss, M. and Hanks, T.: The Source Parameters of the San Fernando Earthquake Inferred from Teleseismic Body Waves, Bull. Seismol. Soc. Am., 62, 591-602, 1972.

Wyss, M. and Molnar, P.: Efficiency, Stress Drop, Apparent Stress, Effective Stress, and Frictional Stress of Denver, Colorado, Earthquakes, J. Geophys. Res., 77, 1433-1437, 1972.

Yoshida, S., Clint, O., and Sammonds, P.: Electric potential changes prior to shear fracture in dry and saturated rocks, Geophys. Res. Lett., 25, 1577-1580, 1998. 